\title{
Coronary venous aneurysms and accessory atrioventricular connections
}

\author{
SIEW YEN HO, * GRAHAM RUSSELL, † EDWARD ROWLAND \\ From the ${ }^{\star}$ Department of Paediatrics, Cardiothoracic Institute, London; $†$ Department of Paediatric \\ Pathology, Bristol Maternity Hospital, Bristol; $\ddagger$ Department of Cardiology, Brompton Hospital, London
}

SUMMARY Histological investigation of a heart with aneurysmal malformation of the anterio $\overrightarrow{\vec{j}}$ cardiac vein showed atypical accessory atrioventricular pathways that could have been the basis fop bypass tracts and ventricular pre-excitation. Review of other cases with coronary sinus or venous aneurysms showed a striking association with sudden death. Such malformations may not be as benign as previously thought.

Aneurysmal malformations of the coronary sinus and coronary veins are not often reported. When present they are casual findings and thought to be of little clinical importance ${ }^{1}$ except in association with ventricular pre-excitation. ${ }^{2}$ We describe a further case with this malformation, which prompted us to re-examine a previous case.

\section{Case report}

The patient, a girl born at Southmead Hospital, presented with a neonatal arrhythmia. The electrocardiogram showed a tachycardia of 200 beats per minute with wide QRS complexes and without obvious $P$ waves. This was interpreted as showing an antidromic tachycardia in association with the Wolff-Parkinson-White syndrome (fig 1). Cardiac catheterisation was performed when she was 11 weeks old. This showed an oval fossa defect with a large right atrium. A curved, band-like chamber below the lower margin of the right atrium was interpreted as being the coronary sinus. The patient suffered a cardiac arrest during this procedure but resuscitation was successful. The parents refused any further investigation. Subsequent medical history was not available. The child died suddenly at the age of 12 .

Necropsy findings limited to the heart showed an

Requests for reprints to Dr Siew Yen Ho, Department of Paediatrics, Cardiothoracic Institute, Fulham Road, London SW3 6HP. enlarged heart weighing $243 \mathrm{~g}$ which had the usua chamber connections and relations with intact septat structures. An aberrant chamber composed of severad
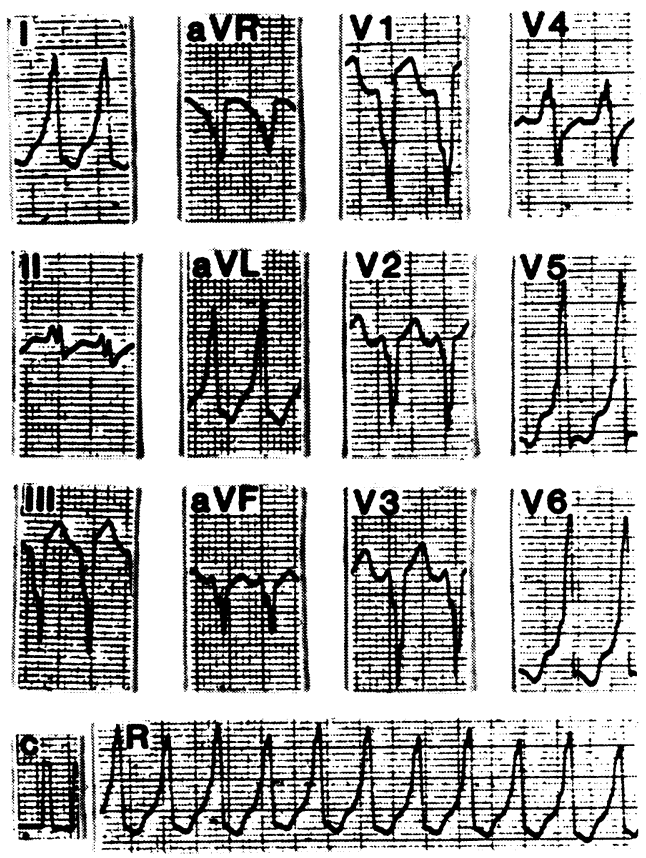

Fig 1 Standard lead electrocardiogram. c, calibration $1 \mathrm{mV} ; R$, rhythm strip. 


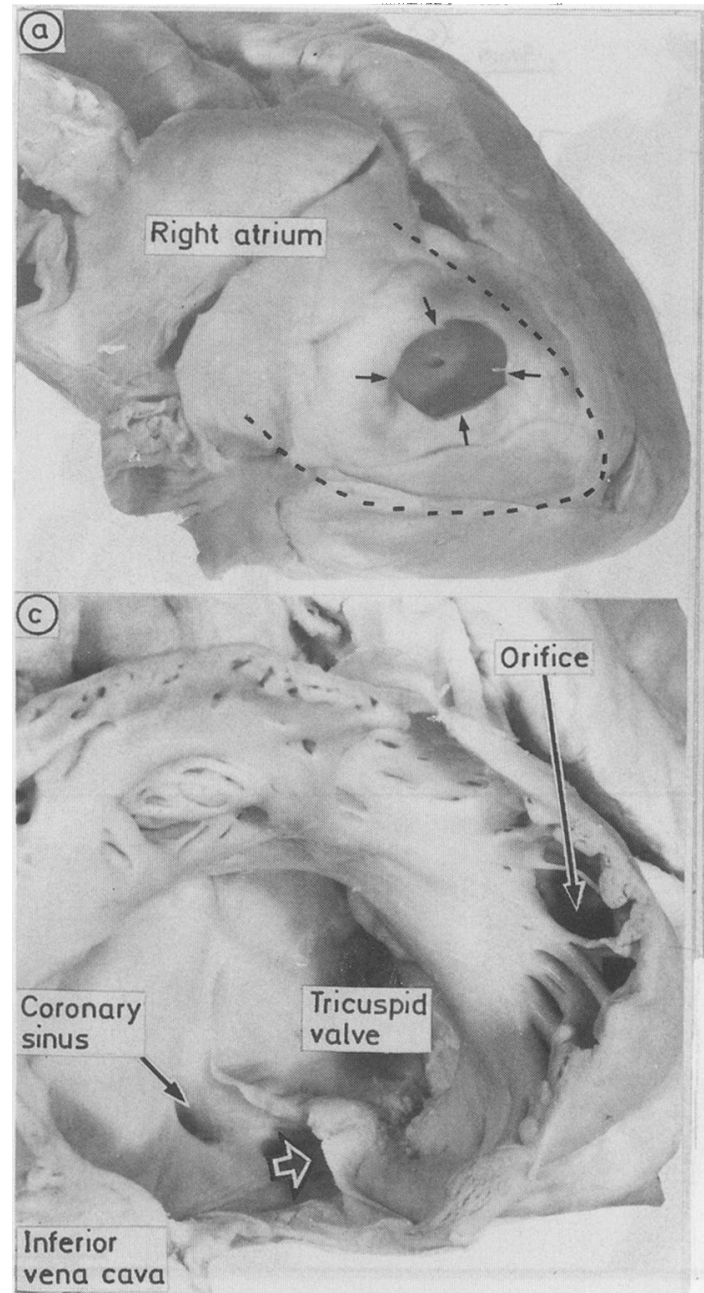

intercommunicating channels separated by trabeculations occupied the position of the right ventricular free wall (fig 2). The chamber had a thin muscular parietal wall. It did not communicate with either the right ventricle or the arterial tree. Instead, it opened directly into the right atrial appendage through an orifice that was located among the atrial trabeculations. The small cardiac vein, which was a continuation of the infundibular vein, ran within atrioventricular groove tissue close to the tricuspid valve. A hypoplastic right coronary artery was present within groove tissue. The aberrant chamber connected with the small cardiac vein anteriorly and became confluent posteriorly just distal to the acute margin. The combined venous orifice opened into

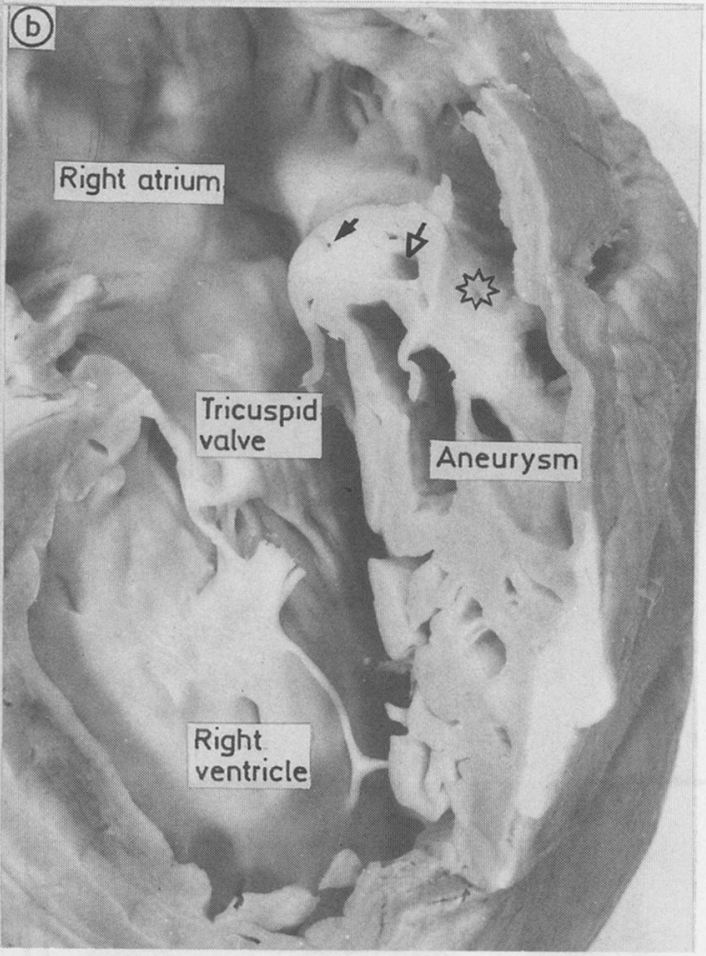

Fig 2 Morphological appearance of the heart (a) viewed from the right to show the extent of the aneurysm which is indicated by the broken line. The arrows point to a window cut in the parietal wall. (b) The right side of the heart viewed from the back after removal of the parietal wall. The atrial orifice (star) to the aneurysm is separated by atrioventricular groove tissue from the tricuspid valve orifice. The small cardiac vein (open arrow) and hypoplastic right coronary artery (closed arrow) are visible. (c) A view into the right atrium showing the atrial orifice of the aneurysm. To the right of the coronary sinus is the posterior venous orifice (open arrow).

the right atrium approximately $1 \mathrm{~cm}$ to the right of the coronary sinus. The coronary sinus orifice was large, and the opening from the posterior interventricular vein was situated in its mouth. Apart from moderate hypertrophy and mild endocardial fibroelastosis of the left ventricle, the rest of the heart was normal.

Histological sections through the venous orifice in the right atrial appendage showed continuity between atrial and ventricular myocardium (fig 3). A 2 $\mathrm{mm}$ wide band of enlarged and aligned myocytes passed from the component of atrial wall which formed the parietal aspect of the orifice into the parietal wall of the aneurysm. There was a second band of myocardial continuity to the medial side of 


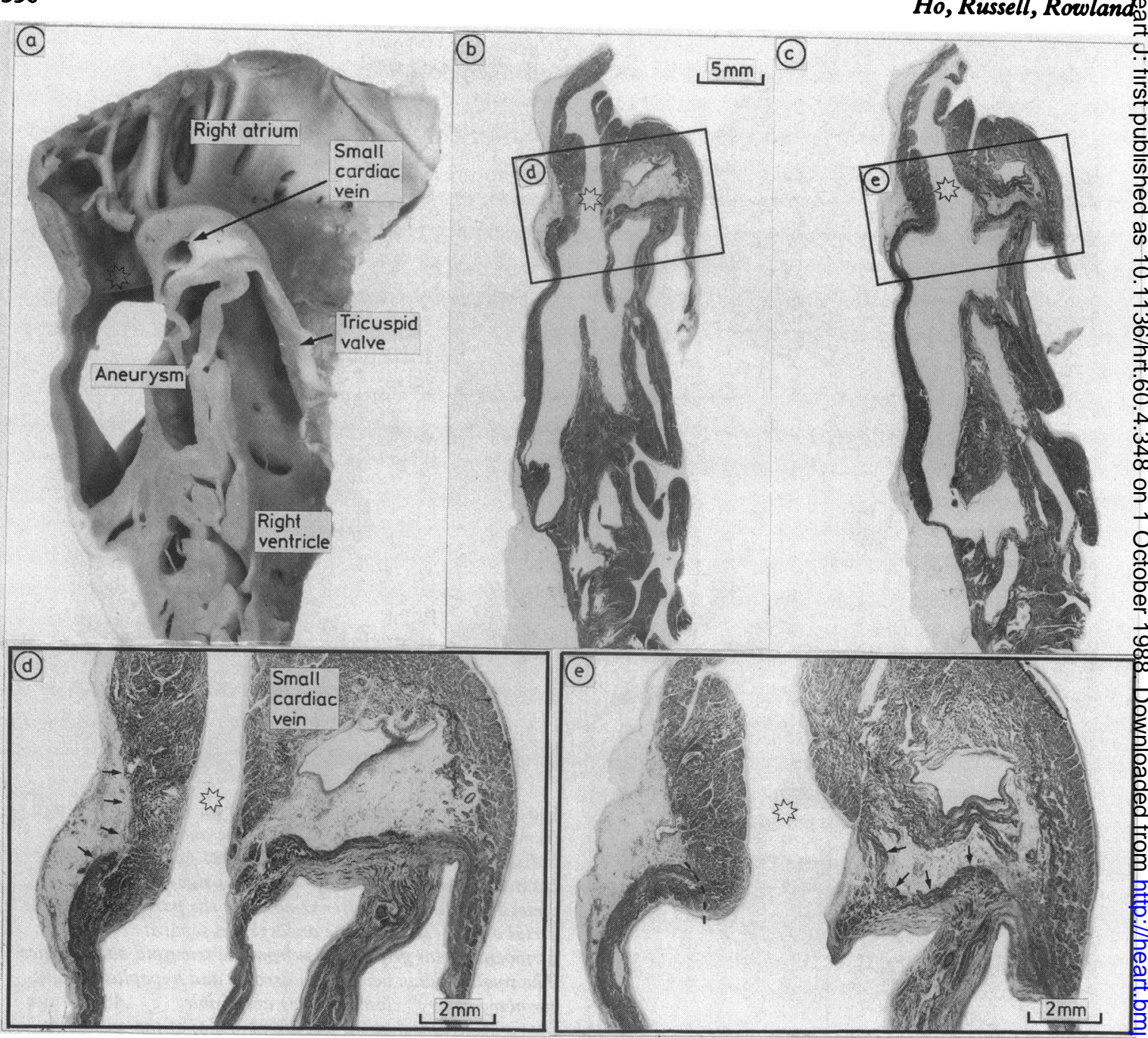

Fig 3 The tissue block removed for histological study is shown in (a). Step sections ( $b$ and $c$ ) with enlargements of the respective boxed areas in $(d)$ and $(e)$. The orifice of the aneurysm is marked by the star in each panel. The accessory connections are indicated by the series of arrows in $(d)$ and $(e)$. Although the parietal wall in $(e)$ shows apparent muscular continuity, there is actually fibrous separation in the area marked by the broken line.

the orifice that ran between the orifice and the small cardiac vein (fig 3 ) before connecting with the party wall between aneurysm and right ventricle.

\section{Discussion}

There are few published reports on the embryogenesis of the human cardiac vein. Studies on the rabbit heart showed that the cardiac veins appeared before the arteries. ${ }^{3}$ The main venous system started as endothelial buds from the coronary sinus. Other outgrowths also developed from the atria and the ventricles. Those from the right atrium may retain their atrial connections to form the anterior cardiac veins or anastomose with the main venous system to open into the coronary sinus. In early development, the atrial and ventricular myocardium is continuous. ${ }^{4} \mathrm{C}$ Continuity around the atrioventricular junction is interrupted by an ingrowth of atrioventriculare groove tissue. ${ }^{5}$ In normal circumstances, muscular continuity is preserved only at the atrioventricular? conduction bundle. Persistence of other myocardial bridges provides the potential for ventricular pre- $\frac{\vec{D}}{\mathbb{N}}$

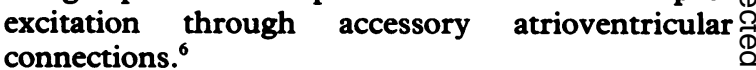

Most accessory atrioventricular connections are 
related to the annular insertions of the atrioventricular valves in the parietal and septal regions. ${ }^{7}$ Infrequently, histological confirmation of atrioventricular connections is found remote from the valve insertions. ${ }^{2}$ Our case is another instance in which myocardial continuity was provided by extensive bands that formed the subepicardial and medial walls of the venous aneurysm. There was inadequate myocardial retrogression ${ }^{4}$ and lack of sequestration by groove tissue in the region of the anterior atrial venous openings. The myocardial bands may be interpreted as persistence of the primitive atrioventricular myocardial connection carried by outgrowing endothelial buds from the right atrium, while the aneurysm is a malformation of the anterior cardiac veins.

The three previous reported patients with venous aneurysms or aneurysms of the coronary sinus died suddenly. ${ }^{12}$ In two of these, there was electrocardiographic evidence of Wolff-Parkinson-White syndrome together with histological demonstration of the accessory pathways. We have reviewed the heart of case 2 that we reported on earlier ${ }^{1}$ (in which there was no evidence of Wolff-Parkinson-White syndrome) and we found potential muscular continuity between atrial and ventricular myocardium through the outer wall of the coronary sinus aneurysm. We believe that this could form the basis for a bypass tract. In view of the alarming association of sudden death with coronary sinus or venous aneurysms, we believe that such malformations may not be as benign as was previously thought. The potential for subepicardial myocardial bridges is enormous because of extensive conjugation between atrial and ventricular myocardium through the outer wall of the aneurysm. These bridges are atypical accessory connections but in our opinion they are easier to ablate than the regular annular types. In our present case both the parietal and medial bands may have been amenable to ablation by cryothermy or dissection around the mouth of the venous aneurysm.

The pattern of pre-excitation seen on the electrocardiogram in this case is consistent with a right sided accessory pathway ${ }^{89}$ which could have been either anterior or posterior. It is possible that, with more extensive sectioning, further accessory connections would have been found. Although it is not possible to be precise about the arrhythmia, it is reasonable to assume that sudden death was caused by atrial fibrillation in Wolff-Parkinson-White syndrome. Because none of the cases with atypical pathways were investigated electrophysiologially, it is not possible to comment on whether the refractory characteristics of the subepicardial pathways were different from pathways that were more adjacent to the cardiac cavities.

We thank Dr S Jordan for the clinical information on this case and Dr L Gerlis for his encouragement throughout this investigation and for preparing fig 1 , and Professor R Anderson for his helpful advice.

\section{References}

1 Ho SY, Gupta I, Anderson RH, Lendon M, Kerr I. Aneurysm of the coronary sinus. Thorax 1983;38: 686-9.

2 Gerlis LM, Davies MJ, Boyle R, Williams G, Scott H. Pre-excitation due to accessory sinoventricular connexions associated with coronary sinus aneurysms. A report of two cases. $\mathrm{Br}$ Heart $J$ 1985;53:314-22.

3 Grant RT. Development of the cardiac coronary vessels in the rabbit. Heart 1926;13:261-71.

4 Walmsley T. The heart. In: Sharpey-Schafer E, Symington J, Bryce TH, eds. Quain's elements of anatomy. London: Longmans, 1929:59-60.

5 Anderson RH, Becker AE. The development of the heart. In: Cardiac anatomy. London: Gower Medical Publishing, 1980:10.23-10.30.

6 Anderson RH, Becker AE, Brechenmacher C, Davies MJ, Rossi L. Ventricular pre-excitation. A proposed nomenclature for its substrates. Eur $J$ Cardiol 1975;3:27-36.

7 Gallagher JJ, Svenson RH, Sealy WC, Wallace AG. The Wolff-Parkinson-White syndrome and the preexcitation dysrhythmias. Medical and surgical treatment. Med Clin North 1976;60:101-23.

8 Gallagher JJ, Pritchett ELC, Sealy WC, Kasell J, Wallace AG. The pre-excitation syndromes. Prog Cardiovasc Dis 1978;30:285-327.

9 Willems JL, Robles de Medina EO, Bernard R, et al. Criteria for intraventricular conduction disturbances and pre-excitation. J Am Coll Cardiol 1985;5:126175. 